Rurali: Revista interdisciplinar de estudios rurales, N. 0, V.1, enero a junio de 2021, pp.18-41 https://doi.org/10.7203/Rurali.1.1.20382

\title{
Los retos de la transformación digital para el derecho de participación política en el mundo rural
}

\section{Digital transformation challenges for political participation in the rural world}

Joaquín Sarrión Esteve*1

\section{Resumen}

La tecnología siempre nos ha acompañado a lo largo de nuestra vida, pero en ocasiones se producen saltos cualitativos de notable relevancia, como sucedió con la imprenta, el barco de vapor, la revolución industrial o las tecnologías de radio, televisión, el ordenador personal, o internet. La transformación digital y todas las tecnologías que conlleva no constituyen por tanto una anomalía en el avance científico-tecnológico, pero sí una nueva revolución que ya está impactando en la forma que tiene el ser humano de aproximarse a la realidad que le rodea, de entender el mundo, e incluso de entenderse a sí mismo y a los demás.

Este trabajo trata de realizar una aproximación -desde la reflexión constitucional- a los retos que plantea esta transformación digital y las tecnologías respecto de la participación política, como derecho, en particular en el mundo rural.

\begin{abstract}
Technology has always accompanied us throughout our lives, but sometimes there are qualitative leaps of notable relevance, as happened with the printing press, the steamship, the industrial or the technologies of radio, television, the personal computer, or Internet. Digital transformation and all the technologies that it entails are therefore not an anomaly ones -in scientific-technological progress-, but a new revolution that is already impacting the way that human beings approach the reality that surrounds them, of understanding the world, and even to understand yourself and others.

The aim of this paper is to focus -from a constitutional reflection- on the challenges posed by this digital transformation and technologies regarding political participation, as a right, particularly in the rural world.
\end{abstract}

\section{Palabras clave}

Transformación digital, participación política, mundo rural, tecnología

\footnotetext{
* Agradecimientos y reconocimientos: Joaquín Sarrión agradece el apoyo de la Ayuda del Programa "Ramón y Cajal" (RYC) 2015, ref. RYC-2015-18821 (FSE/AGENCIA ESTATAL DE INVESTIGACIÓN). Asimismo, agradece los comentarios y observaciones recibidas por los editores y evaluadores de la Revista, que han ayudado a mejorar el texto del trabajo, todos los errores que permanecen corresponden al autor.

${ }^{1}$ Investigador Ramón y Cajal. Departamento de Derecho Constitucional. Universidad Nacional de Educación a Distancia (UNED). jsarrion@der.uned.es
} 


\section{Key words}

Digital transformation, political participation, rural world, technology

Sumario.- 1. Motivación. 2. Breve aproximación al marco constitucional de la participación política. 3. La e-participación como instrumento de participación política en un mundo digital. 4. La e-participación en las redes: del estatus de consumidor al estatus de ciudadano. 5. El gobierno inteligente y la e-participación. 6. A modo de conclusión: ¿Hacia una democracia digital?. 7. Recursos bibliográficos.

\section{Motivación}

La tecnología siempre nos ha acompañado a lo largo de nuestra vida, pero en ocasiones se producen saltos cualitativos de notable relevancia, como sucedió con la imprenta, el barco de vapor, la revolución industrial o las tecnologías de radio, televisión, el ordenador personal, e internet. La transformación digital y todas las tecnologías que conlleva no constituyen por tanto una anomalía en el avance científico-tecnológico, pero sí una nueva revolución que ya está impactando en la forma que tiene el ser humano de aproximarse a la realidad que le rodea, de entender el mundo, e incluso de entenderse a sí mismo y a los demás.

Estas tecnologías, en particular las vinculadas con la digitalización, permiten que el ciudadano tenga un mayor acceso a información de los asuntos públicos y a debatir en la nueva ágora que suponen las redes sociales. Casi todos los días podemos encontrar encuestas que se lanzan en las diferentes redes, por parte de periodistas, empresas, cadenas de televisión, o incluso particulares que utilizan las redes.

Las ventajas de la participación a través de medios electrónicos, y las plataformas creadas en el mundo digital, son evidentes; siempre que se garantice el acceso de todos los ciudadanos a los mismos, y estos se sujeten a principios de neutralidad en el debate público, lo que no son ni pocas cosas, ni tampoco sencillas. Por un lado, no todos los ciudadanos (aunque sí una gran mayoría) tienen la capacidad económica para poder adquirir y mantener instrumentos de acceso a las redes, la cobertura necesaria para ello, y el tiempo libre para dedicarlo a estos menesteres; a la vez que los medios -con carácter general- nacen de la iniciativa privada, y por tanto son empresas que responden a sus propios intereses y objetivos.

Si la participación electrónica o lo que podemos denominar e-participación puede ser un buen instrumento para fomentar y facilitar la participación de los ciudadanos en la esfera pública, para expresar su opinión y valorarla de forma adecuada, no es menos cierto que no deja de suponer una nueva plaza pública virtual, con los peligros intrínsecos que esto conlleva, tanto en lo relativo a la gobernanza inteligente (Smart Governance) que facilita la 
participación ciudadana en la gestión de los asuntos públicos, como incluso dentro de un concepto más amplio que abarque la participación política de los ciudadanos en un sentido mucho más amplio, la democracia electrónica o digital, es decir, la e-democracia ${ }^{2}$.

En este trabajo vamos a realizar una breve aproximación al marco constitucional sobre la participación política, para posteriormente analizar la participación digital o electrónica (e-participación) como instrumento de participación política en un mundo en plena transformación digital o, mejor dicho, en un mundo ya digital.

\section{Breve aproximación al marco constitucional de la participación política}

La Constitución española de 1978 (en adelante CE) consagra la participación política en el art. $23^{3}$, si bien, en realidad, se puede decir que es el primer apartado el que regula de una forma general el derecho de participación, y el apartado segundo recoge de forma específica el derecho de acceso a cargos y funciones públicas en condiciones de igualdad, no siendo este último sino una manifestación específica del primero o, para ser más exactos, una garantía de que la participación directa en los asuntos públicos a través del acceso a las funciones y cargos públicos se pueda realizar en igualdad de condiciones, por parte de los ciudadanos, conforme a los requisitos establecidos legalmente.

No agota, ni mucho menos, el art. 23, la cobertura constitucional a la participación política, sino que podríamos decir que este derecho "se diversifica" (Gómez Sánchez, 2018: 511), y podemos encontrarlo en la regulación de los partidos políticos como instrumento de participación política (art. 6), la previsión de los poderes públicos promociones la participación en (art. 9), la participación en educación (art. 27.5), el derecho de petición (art. 29), el derecho de participación de la juventud (art. 48), las peticiones individuales o colectivas a las Cámaras parlamentarias (art. 77), la iniciativa legislativa popular (art. 87.3), el referéndum (art. 92) ${ }^{4}$, el derecho a ser oído en ciertos procedimientos (art. 105), la participación en la Administración de Justicia mediante la acción popular y la institución del Jurado (art. 125), la participación en la Seguridad Social y en la empresa (art. 129); si bien hay una clara opción por el sistema representativo, al regular de forma escasa las otras modalidades, y consagrar a los partidos políticos como "instrumentos fundamentales para la participación política"5. Y ello sin perjuicio de que otros derechos, en algunos casos fundamentales, puedan también dar cobertura a la participación política, como puede ser, sin ir más lejos, con la libertad de expresión (art. 20.1 a) y la libertad de información (art. 20.1 c) ${ }^{6}$.

\footnotetext{
${ }^{2}$ Véase Pérez Zafrilla et al (2021).

${ }^{3}$ «1. Los ciudadanos tienen el derecho a participar en los asuntos públicos, directamente o por medio de representantes, libremente elegidos en elecciones periódicas por sufragio universal./2. Asimismo, tienen derecho a acceder en condiciones de igualdad a las funciones y cargos públicos, con los requisitos que señalen las leyes.»

${ }^{4}$ Hay que considerar el referéndum consultivo (art. $92 \mathrm{CE}$ ), y el referéndum de reforma constitucional (arts. 167.3 y $168.3 \mathrm{CE})$.

${ }^{5}$ Véase Gómez Sánchez (2018:512).

${ }^{6}$ No hace falta subrayar la intrínseca y relevante conexión existente entre la libertad de expresión, así como el derecho a comunicar o recibir una información veraz, con la democracia y la participación política.
} 
Cabe diferenciar desde un punto de vista jurídico diferentes tipos o tipologías de participación política. Así, podemos hablar de participación directa o indirecta (o representativa, es decir, a través de representantes). Dentro de la participación representativa hay que considerar de forma particular el sufragio activo y pasivo. Asimismo, la participación política, sea directa o indirecta, también se puede diferenciar en función del ámbito de su realización, tanto a nivel interno (nacional, regional, municipal), pero también a nivel europeo.

En efecto, también en la Unión Europea encontramos de una forma participar pues el Tratado de la Unión Europea consagra la democracia representativa (art. 10 TUE), así como también el derecho de los ciudadanos "a participar en la vida democrática de la Unión", incluso incidiendo que la toma de decisiones será de la forma más abierta y próxima posible, pasando por el importante instrumento de la iniciativa ciudadana europea (art. 11.4 TUE) ${ }^{7}$.

Sin embargo, lo que nos interesa no es tanto la regulación constitucional y de la Unión Europea, sino una perspectiva diferente: en qué medida las tecnologías disruptivas pueden potenciar o afectar a la participación política el mundo rural.

\section{La e-participación como instrumento de participación política en un mundo digital}

Sin duda, como hemos anticipado, las nuevas tecnologías permiten que el ciudadano tenga un mayor acceso a la información de los asuntos públicos y a debatir en la nueva ágora de las redes sociales.

Se suele poner el acento en la Smart Governance como estrategia de un tipo de gobierno inteligente para fomentar la participación en la gestión pública, mejorando la transparencia pública en las Smart Cities (Santiago Iglesias, 2018: 414), pues como posteriormente veremos, es un fenómeno fundamentalmente local o municipal, y que pone el acento en la ciudad o la urbe, también porque normalmente son las ciudades grandes las que por un lado tienen los recursos para implementar estos instrumentos de gobernanza digital más activos y, por otro lado, no tienen la posibilidad de reunir a los vecinos en la plaza del pueblo como ocurría en una pequeña localidad del mundo rural vaciado.

Sin embargo, debemos pensar de forma más ambiciosa cuando nos aproximamos a la e-participación, o a la participación utilizando medios electrónicos, pues forma parte de la transformación digital que estamos viviendo gracias a las tecnologías disruptivas que no hacen sino progresar de una forma cada vez más acelerada y convertir nuestra realidad en una realidad digital, en una vida en un mundo digital; o como se ha dicho, the onlife world (Hildebrant, 2016: 45).

En efecto, en la actualidad vivimos inmersos en un proceso de transformación digital en el que cada vez adquirieren una mayor relevancia las llamadas "tecnologías disruptivas inteligentes" o simplemente tecnologías inteligentes (Smart technologies), que están

\footnotetext{
${ }^{7}$ Cuya regulación se complementa en el art. 24 del Tratado de Funcionamiento de la Unión Europea (TFUE), y en el Reglamento 211/2011 del Parlamento Europeo y del Consejo de 16 de febrero de 2011, y el Reglamento de Ejecución 1179/2011, de la comisión, de 17 de noviembre de 2011. Sobre la iniciativa ciudadana europea y sus particularidades se puede acudir a Gómez Sánchez (2013) y Burguera Ameave (2014).
} 
afectando al mundo del Derecho en general -hasta el punto de que se comienza a hablar del Derecho digital como una disciplina autónoma dentro del Derecho (Barrio Andrés, 2020), y en particular a los derechos fundamentales, al menos tal y como estaban concebidos hasta la actualidad, junto con las garantías establecidas para los mismos (Sarrión Esteve, 2020a: 322), incluyendo, por supuesto, el derecho participación política, o la nueva e-participación.

Hasta tal punto esto es así que esta transformación plantea serios desafíos para el Derecho, y para sus fines ${ }^{8}$, también para los derechos fundamentales, a los que nos vamos a enfrentar en cualquier esfera de nuestra vida, y que conllevan un serio potencial de exclusión digital y otros menoscabos en los derechos. Esto cobra especial importancia si tenemos en cuenta el diferente acceso a las tecnologías digitales del mundo rural frente a la ciudad (de hecho, se suele hablar de Smart cities, pero no de Smart rurality), y la mayor parte de nuestro territorio, donde se concentra una parte importante, aunque no mayoritaria de la población, es esencialmente rural.

En primer lugar, es importante identificar correctamente y delimitar las tecnologías que integran las Smart Technologies, cuyos elementos de identificación vendrían determinado por los conceptos que integran la expresión, esto es, "tecnología" (instrumentos y procedimientos industriales) ${ }^{9}$ e "inteligencia" (que implica capacidad de entender o comprender, pero también capacidad de resolver problemas, esto segundo sí ha sido alcanzado ya por ciertas tecnologías disruptivas) ${ }^{10}$; por lo que podríamos considerar como tales: la Inteligencia Artificial (IA), los robots y la robotización de los sistemas (hardware y software), cadenas de bloques (Blockchain), el Internet de las Cosas (o más conocida en inglés como Internet of Things, IoT), la utilización o tratamiento masivo de datos (o Big Data), los sistemas de decisión automatizados, sistemas de reconocimiento (facial, voz, etc.),

\footnotetext{
${ }^{8}$ De nuevo, Hildebrandt (2016)

${ }^{9}$ Conforme al Diccionario de la Real Academia Española (RAE), tecnología es:

"1. f. Conjunto de teorías y de técnicas que permiten el aprovechamiento práctico del conocimiento científico.

2. f. Tratado de los términos técnicos.

3. f. Lenguaje propio de una ciencia o de un arte.

4. f. Conjunto de los instrumentos y procedimientos industriales de un determinado sector o producto". Accesible en: https://dle.rae.es/?id=ZJ2KRZZ (Último acceso 13.09.2019)

${ }^{10}$ Conforme al Diccionario de la RAE, inteligencia es:

“1. f. Capacidad de entender o comprender.

2. f. Capacidad de resolver problemas.

3. f. Conocimiento, comprensión, acto de entender.

4. f. Sentido en que se puede tomar una proposición, un dicho o una expresión.

5. f. Habilidad, destreza y experiencia.

6. f. Trato y correspondencia secreta de dos o más personas o naciones entre sí.

7. f. Sustancia puramente espiritual.

8. f. servicio de inteligencia. inteligencia artificial

1. f. Inform. Disciplina científica que se ocupa de crear programas informáticos que ejecutan operaciones comparables a las que realiza la mente humana, como el aprendizaje o el razonamiento lógico.

(...)" Accesible en: https://dle.rae.es/?id=LqtyoaQ/LqusWqH (Último acceso 13.09.2019).
} 
el machine learning (o en el futuro el machine teaching) ${ }^{11}$, o también los drones (así los drones autónomos) $)^{12}$, etc...

En segundo lugar, hay que analizar qué función le corresponde asumir al Derecho, y en particular al Derecho Público, y dentro del mismo al Derecho Constitucional, respecto a estas tecnologías y lo que suponen, y que necesariamente debe implicar por un lado atender a las fases de su investigación, diseño, desarrollo, implementación, así como también su utilización, haciendo referencia a las diferentes esferas, campos o temas en los que se pueden utilizar: seguridad pública y privada, sanidad, medicina, investigación, servicios, y por supuesto, lo que más nos interesa: la participación política.

Desde luego, no es una preocupación que surja ahora, por un lado EEUU (Weller, 2016) o incluso antes en Japón (Christensen, 2006), donde llevan ya bastante tiempo estudiando la necesidad de adaptar la legislación a la IA y la robótica, adaptando sus marcos normativos para potenciar la investigación y desarrollo de estas tecnologías; y la Unión Europea ha tomado nota del reto que suponen, así, el Parlamento Europeo aprobó la Resolución de 16 de febrero de 2017 sobre la necesidad de elaborar una normativa civil a nivel europeo sobre robótica (Resolución sobre normas de Derecho civil sobre robótica), en la que se reflejen «los valores humanistas intrínsecamente europeos y universales que caracterizan la contribución de Europa a la sociedad» (Parlamento Europeo, 2017).

Por su parte la Comisión Europea emitió una Comunicación sobre "Inteligencia artificial para Europa" en la que muestra la necesidad de adherirse al cambio que supone el advenimiento de la IA (Comisión Europea, 2018a). El Informe del Joint Research Centre Artificial Intelligence. A European Perspective (Craglia, 2018) muestra de una forma muy desarrollada la perspectiva europea -predominantemente antropocéntrica- sobre la Inteligencia Artificial. Recientemente, el Parlamento Europeo ha adoptado la Resolución de 12 de febrero de 2019 sobre "Una política industrial global europea en materia de inteligencia artificial y robótica" (Parlamento Europeo, 2019).

También es importante resaltar la Propuesta de Reglamento por el que se establece el programa Europa Digital para el período 2021-2027 actualmente en fase de primera lectura tanto en el Parlamento Europeo como el Consejo (Comisión Europea, 2018b). Así como la constitución del Grupo Independiente de Expertos de Alto Nivel sobre Inteligencia Artificial de la Comisión Europea (High-Level Expert Group on Artificial Intelligence, AI HLEG) en junio de 2018 con el propósito de apoyar la implementación de la Estrategia Europea en Inteligencia Artificial, y que ha tenido ya la oportunidad de desarrollar las "Directrices Éticas para una IA Fiable" (Grupo Independiente de Expertos de Alto Nivel sobre Inteligencia Artificial de la Comisión Europea, 2019), con una aproximación antropocéntrica, así como

\footnotetext{
${ }^{11}$ Aunque actualmente hay un gran desarrollo del machine learning, entendido como que las máquinas, robots o programas son capaces de aprender por ellos mismos, no es descartable que en un futuro se ocupen también de enseñar al ser humano (machine teaching).

${ }^{12}$ Se entiende por drones los vehículos aéreos no tripulados, incluyendo aquellos que son pilotados de forma remota (RPA) o aquellos que tienen un pilotaje autónomo, en cuyo caso sí podríamos incluirlos dentro de las tecnologías inteligentes. Sobre los retos que plantean los drones, véase Sarrión Esteve y Benlloch Domènech (2017).
} 
se ha lanzado la Alianza Europea en IA (European AI Alliance) que tuvo su primera asamblea en junio de 2019 y que próximamente tendrá su segunda asamblea en $2020^{13}$.

Asimismo, es importante tener en consideración el LIBRO BLANCO sobre la inteligencia artificial - un enfoque europeo orientado a la excelencia y la confianza, disponible en español, de la Comisión Europea, publicado el 19 de febrero de 2020, y que establece una serie de garantías y limitaciones al uso de algunas tecnologías de la Inteligencia Artificial (Comisión Europea, 2020a) así como el Informe sobre implicaciones de la Inteligencia Artificial, el Internet de las Cosas y la robótica, que se ha presentado (Comisión Europea, 2020b).

Por su parte, en el marco del Consejo de Europa, se ha subrayado la complejidad y naturaleza envolvente de estas tecnologías emergentes que están planteando serios retos a la regulación y también a los derechos humanos llamando a una mayor cooperación a nivel internacional para afrontar los retos de estas tecnologías ${ }^{14}$, en cuyo marco, en la Comisión europea para la eficacia de la justicia (CEPEJ), se ha elaborado la Carta Europea Ética sobre el Uso de la Inteligencia Artificial en los sistemas judiciales y su ámbito (Consejo de Europa, 2018), así como se ha creado un Comité de Expertos sobre las dimensiones de los derechos humanos del tratamiento masivo de datos y las diferentes formas de Inteligencia Artificial (Committee of experts on human rights dimensions of automated data processing and different forms of artificial intelligence), que ya ha tenido oportunidad de elaborar diversos trabajos de interés.

A nivel nacional, se ha aprobado recientemente la Estrategia Española de I+D+I en Inteligencia Artificial (Ministerio de Ciencia, Innovación y Universidades, 2019).

Además, y aunque no es el objeto de este trabajo, no se puede dejar de señalar que se ha comenzado a dar importancia a la utilización de la geolocalización por apps y smartphones, en España, con ocasión de la crisis sanitaria que vivimos por la pandemia del COVID-19, lo que ha motivado que dentro de las medidas adicionales a la declaración del estado de alarma, se dictara la Orden SND/297/2020, de 27 de marzo, por la que se encomienda a la Secretaría de Estado de Digitalización e Inteligencia Artificial, del Ministerio de Asuntos Económicos y Transformación Digital, el desarrollo de diversas actuaciones para la gestión de la crisis sanitaria ocasionada por el COVID-19, que incluyen el desarrollo de soluciones tecnológicas y aplicaciones móviles para la recopilación de datos para la mejora de la eficiencia operativa de los servicios sanitarios y una mejor atención y accesibilidad a los mismos por los ciudadanos, así como también encomendar un estudio de la movilidad aplicada a la crisis sanitaria (DataCOVID-19), todo ello sin perjuicio de la aplicación del régimen de protección de datos ${ }^{15}$, y que ha posibilitado la creación y desarrollo de la aplicación "Radar COVID". De hecho, la comunidad científica ha sido consciente de la necesidad de transparencia de que se hiciera público un repositorio del código para analizar los elementos del sistema, que incluya el historial y cambios, informe de diseño del

\footnotetext{
${ }^{13}$ Véase https://ec.europa.eu/futurium/en/eu-ai-alliance (Último acceso 19.09.2020).

${ }^{14}$ Véase el Informe elaborado por Déaut (2017), la recomendación de la Asamblea del Consejo de Europa y la contestación del Consejo de Ministros (Consejo de Europa, 2017a y 2017b).

${ }^{15}$ Sobre esta cuestión, véase el trabajo de Ricard Martínez (2020).
} 
sistema, informe detallado de los mecanismos de monotorización, y una evaluación de impacto en la protección de datos (Gonzalo, 2020).

Desde luego, la doctrina iuspublicista, incluyendo la constitucional, ha puesto el foco y atención en estos retos a nivel nacional ${ }^{16}$ y, en Abril de 2017, se constituyó la Red Derecho Administrativo e Inteligencia Artificial. En este ámbito se ha organizado el I Seminario Internacional Derecho Administrativo e Inteligencia Artificial (DAIA) ${ }^{17}$, que posteriormente ha tenido desarrollo y continuidad. Estamos ante una atención que se une a la que ya se había comenzado a dispensar en la literatura a nivel internacional unos años antes.

Sin duda, los retos que suscitan estas tecnologías para el Derecho Público requiere que avancemos en el estudio de los múltiples problemas que se plantean, y requeriría de una labor multidisciplinar, incluyendo al Derecho (y sus distintas áreas), pero no sólo; y es que hay que concretar las diferentes narrativas de la inteligencia artificial (López Baroni, 2019) para poder centrar el foco de nuestro análisis, y sin duda algunas recientes obras colectivas están en ese camino (Barrio Andrés, 2018; García Novoa et al, 2018; Pérez Zafrilla et al, 2021).

En esta última obra ${ }^{18}$, se plantea precisamente que. desde una perspectiva muldisciplinar, la respuesta jurídica frente a los retos que la Inteligencia Artificial plantea será más efectiva. Esto se puede afirmar con carácter general, pues: 1) hay una clara necesidad de establecer un concepto autónomo de Inteligencia Artificial que nos permita delimitar esta tecnología de las otras tecnologías inteligentes que están emergiendo; 2) y también una necesidad de regular la investigación, diseño, implementación y utilización de sistemas que incorporen inteligencia artificial, lo que además de en general, es aplicable también de forma particular a la participación política, y la e-participación (Pérez Zafrilla et al, 2021: 114)

Esto se pone de manifiesto con otra cuestión, no es sólo el posible y eventual uso de la IA sobre los derechos, incluyendo sobre el derecho de participación política y sus diferentes formas a través de procedimientos electrónicos, sino también teniendo en consideración el avance y progresiva convergencia de la IA con el resto de tecnologías inteligentes (Smart Technologies) incluyendo el Blockchain ${ }^{19}$, pero también otras como los drones (Sarrión Esteve, 2018), el Big Data, etc., crea la necesidad de una aproximación también convergente a las mismas, puesto que están transformando nuestra realidad de una forma sustancial afectando también al paradigma de los derechos fundamentales, y es que estas tecnologías van a afectar a las personas en cualquier esfera de su vida (educación, integridad, privacidad, imagen, igualdad, consumo, etc.) y por tanto también a la participación política; y pueden conllevar un serio potencial de exclusión digital y otros

\footnotetext{
${ }^{16}$ Véase los trabajos, por ejemplo, y sin pretensión de exhaustividad de Asís Roig (2014), Sánchez Barrilao (2016), Cotino Hueso (2017), Barrio Andrés (2018), Valero Torrijos (2019).

${ }_{17}$ Véase las Conclusiones del I Seminario de Internacional Derecho Administrativo e Inteligencia Artificial. DAIA (2019).

${ }^{18}$ En particular, en los capítulos 4 y 5 (Pérez Zafrilla et al, 2021: 103 y ss.)

${ }^{19}$ Véase a este respecto a Pollock (2018), Wolfson (2018), y Banafa, que plantea que aunque Blochchain e Inteligencia Artificial son dos campos tecnológicos distintos hay unas grandes posibilidades de obtener aplicaciones derivadas de su convergencia (Banafa, 2019).
} 
menoscabos en los derechos (Sarrión Esteve, 2018), lo que sería especialmente relevante respecto de la participación política en el mundo rural.

Estas tecnologías constituyen, sin duda, algunos de los grandes avances científicos que requieren de un marco legal adecuado que garantice al mismo tiempo el avance de la investigación científico-tecnológica para una utilización eficiente y segura, pero también con el máximo respeto de los derechos fundamentales (tanto en el diseño como en su utilización), y esto requiere, sin duda, una necesaria reflexión desde el Derecho, y en particular desde Derecho Constitucional, teniendo en cuenta que podemos estar ante la evolución o nacimiento de un nuevo paradigma en la teoría general de los derechos fundamentales (Sarrión Esteve, 2020b).

\section{a) Una aproximación jurídica y ética común a las tecnologías convergentes}

Dada la convergencia de las tecnologías, entiendo que frente a las actuales aproximaciones parciales a las distintas tecnologías sería conveniente, al menos desde una perspectiva de análisis ético y jurídico, aproximarnos a ellas teniendo en consideración que podemos identificar unos problemas éticos y jurídicos comunes, aplicables y exigibles tanto en a la hora de regular la investigación, diseño, implementación y utilización de estas tecnologías, garantizando en todo momento que se cumplen las exigencias éticas, de valores, y desde luego los eventuales derechos fundamentales que se pueden ver afectados.

Sin duda, estas tecnologías van a tener una gran aplicación -aunque ya se estén utilizando en la práctica en algunos- en todos los sectores, pasando desde el uso militar hasta el uso civil en distintos campos como la propia Administración Pública, el transporte, el sector bancario, relaciones laborales, el mundo del seguro, sanidad, educación, etc. por poner algunos ejemplos en los que podemos pensar. Desde luego, aquí y ahora nos interesa analizar cómo pueden impactar en la participación política, y en qué medida pueden definir lo que es y será en el futuro la e-participación.

La investigación con una singular tecnología inteligente (IA, blockchain, robots, IoT., etc.) por sí misma o de forma combinada con otras -por su convergencia- debería conllevar ya la exigencia de que dicha investigación, como el posterior diseño de los sistemas, implementación y su posterior utilización en el campo específico responda a las exigencias éticas y legales que garanticen el máximo respeto de los valores éticos (comunes) y derechos fundamentales, incluyendo garantizar la e-participación de todos los ciudadanos, en condiciones de igualdad.

Desde luego para una aproximación de estas características requerimos de establecer un concepto de estas tecnologías apropiado, lo que no es sencillo. Y es que estamos ante un sector que está creciendo a pasos agigantados, y que por sus características se hace difícil llevar a cabo la tarea: IA, robótica, gestión de los sistemas de Big Data, el Internet de las cosas, drones autónomos, vehículos autónomos, etc.; pero es necesario delimitarlas correctamente para, por un lado estudiar la regulación actualmente existente, y por otro lado ver, en qué medida existen retos ya hoy en día no resueltos y que requieren propuestas de lege ferenda. 
Además, el Derecho público debe dar un concepto propio autónomo ${ }^{20}$, aunque parta de la ayuda que le pueden proporcionar otras disciplinas, como la Filosofía o la Sociología, y aunque sea de carácter provisional y susceptible de evolución con las propias tecnologías, porque de otra forma es muy difícil garantizar las exigencias éticas, y en particular la protección de los derechos fundamentales, tanto en la investigación y diseño, como también en su desarrollo e implementación; únicamente de esta forma se podrá garantizar -a la largo plazo- la prevención de los desafíos que se plantean, lo que incluye la participación política, y los eventuales efectos e influencias que estas tecnologías pueden tener para la democracia.

La IA, entendida como la innovación que permite dotar de autonomía y capacidad a un sistema para desarrollar tareas que exigen inteligencia ${ }^{21}$, está presente en los smart robots o robots inteligentes ${ }^{22}$, en los sistemas de reconocimiento de voz, instrumentos de seguridad se smartphones, traductores, gestores de contenidos audiovisuales digitales, sistemas de reconocimiento de imágenes, o, en general los instrumentos o sistemas computacionales con toma de decisiones automatizadas utilizando Big Data.

El Parlamento Europeo es consciente de que los macrodatos (Big Data) pueden capacitar dispositivos de IA a modo de redes neuronales y modelos estadísticos que sirvan para predecir algunos acontecimientos y comportamientos (Parlamento Europeo, 2017), y estos sistemas computacionales van a ser capaces de tratar, aprender y resolver problemas, tomando decisiones a partir de Big Data, con un cambio de paradigma en cómo se tomaban las decisiones hasta el momento (Cotino Hueso, 2017:132), puesto que los datos y recomendaciones o propuestas propiciados por estos sistemas van a ser fundamentales o incluso determinantes, siendo difícil, que un operador humano se aparte de la recomendación dada por un sistema inteligente salvo casos excepcionales; y dado que en otras ocasiones, directamente las decisiones van a ser automatizadas.

Estos avances, si bien pueden ser vistos de un modo positivo en general, preocupan en relación a su posible uso con fines maliciosos, de influencia y determinación de los resultados electorales. Sin ir más lejos, está muy reciente el escándalo del caso de Facebook y Cambridge Analytica, cuando se destapó que a través de un test de personalidad se pudieron recolectar datos de usuarios que se vendieron a la empresa a efectos de su uso para influenciar resultados electorales, tanto en las elecciones presidenciales de EEUU en 2016, como en el referéndum del Brexit del mismo año, y todo ello sin el consentimiento explícito de los afectados ${ }^{23}$.

Esto tenemos que conectarlo con el avance del IoT; y la evolución del machine learning -como aprendizaje automático - al deep learning - aprendizaje profundo- (Vida Fernández, 2018: 212), cuando es la propia tecnología la que aprende por sí sola. Y por qué no, quizá en un futuro no muy lejano el machine teaching, es decir, cuando es la tecnología

\footnotetext{
${ }^{20}$ En este sentido, y respecto a la IA se pronuncia Sánchez Barrilao (2016).

${ }^{21}$ Una definición bien desarrollada es la que se identifica en la Comunicación de la Comisión Europea "Inteligencia artificial para Europa", en el sentido de identificar a "los sistemas que manifiestan un comportamiento inteligente, pues son capaces de analizar su entorno y pasar a la acción -con cierto grado de autonomía- con el fin de alcanzar objetivos específicos" (Comisión Europea, 2018a).

${ }^{22}$ Los robots, o la robótica en general, cabe distinguirla de la IA, porque no van a integrar necesariamente IA.

${ }^{23}$ Véase Cambridge Analytica: la multa récord que deberá pagar Facebook por la forma en que manejó los datos de 87 millones de usuarios (2019).
} 
la que enseña al ser humano (Pérez Zafrilla et al, 2021: 119). Desde luego debemos considerar como factible que en un futuro no muy lejano la tecnología pueda ser considerada como inteligente, y pueda en ese sentido suponer un salto cualitativo ${ }^{24}$.

\section{b) Hacia un nuevo paradigma en la protección de los derechos fundamentales.}

Ciertamente una cuestión a resolver es identificar los derechos fundamentales que se pueden ver afectados por las tecnologías inteligentes (dignidad, vida, integridad física, igualdad, privacidad, imagen, protección de datos, educación, salud, etc.) entre los que se incluye el derecho de participación política, la e-participación, lo que exigiría por un lado analizar las eventuales utilizaciones de estas tecnologías y su posible repercusión en los distintos derechos fundamentales, y en el derecho fundamental en particular; lo que llevaría por otro lado a la necesidad de delimitar su contenido y garantías necesarias.

Sin embargo, también hay que contestar a una pregunta previa, y es si se puede mantener el paradigma de los derechos fundamentales o es necesario una reorientación a consecuencia de la naturaleza de las tecnologías de las que hablamos.

Algunos autores han sugerido que es necesario abordar la protección de los derechos fundamentales en relación estas tecnologías vinculadas a la IA con nuevas regulaciones basadas en la dimensión colectiva de los derechos ya que estamos hablando de una tecnología que afecta a la humanidad colectivamente, por lo que no podemos simplemente utilizar una dimensión individual o subjetiva (Cotino Hueso, 2017: 137).

También es evidente que es necesario abordar la cuestión de la titularidad, es decir, si los derechos solo deberían proteger a los seres humanos de la IA, o bien también deberían proteger el producto de la IA, como, por ejemplo, si el contenido generado por los sistemas automatizados estaría protegido por la libertad de expresión (Cotino Hueso, 2017: 137); o la protección de los mismos sistemas cuando incorporan una entidad física como un robot en forma humana, como Darling ha propuesto para los robots sociales, de la misma manera que se ha otorgado cierta protección a los animales en la medida en que puedan generar empatía (Darling, 2016), o que han sido defendidos en otros campos sobre la base de la apariencia humana que pueden adquirir y para evitar lo que podría considerarse una forma de esclavitud (Lumpkin, 2013); aunque, como se ha argumentado, los robots deberían, para que se les otorguen ciertos derechos, y por lo tanto una identidad propia, disfrutar de una mayor autonomía y una mayor capacidad de razonamiento que la que tienen actualmente (Replicante Legal, 2014). También cabe plantearse la necesidad o conveniencia de reconocer nuevos derechos o garantías.

Es cierto que, a nivel de la Unión Europea, el Reglamento General de Protección de Datos (RGPD), aplicable desde el 25 de mayo de 2018, ya tiene en consideración algunas de los retos que ha planteado el Big Data y las tecnologías inteligentes. Asimismo, en España se ha utilizado como fuente de implementación y adaptación a las reformas introducidas la

\footnotetext{
${ }^{24}$ Por ello es manifiesto el interés de la Comisión Europea en lograr una adecuada regulación de la Inteligencia Artificial (Comisión Europea, 2021). Esta propuesta ha sido presentada con posterioridad al desarrollo de este trabajo; para un primer análisis de la misma se puede acudir Huergo Lora (2021).
} 
Ley Orgánica 3/2018, de 5 de diciembre, de Protección de Datos Personales y garantía de los derechos digitales (en adelante LODPGDG), con entrada en vigor el 7 de diciembre de 2018, cuyo objetivo es en realidad doble: en primer lugar pretende la adaptación del ordenamiento jurídico español al RGPD y por tanto desarrollar la protección de datos de carácter personal en España; pero también pretende, en segundo lugar, garantizar los derechos digitales de la ciudadanía, en cuanto que nuevos derechos que serían ejercitables frente a la transformación digital ${ }^{25}$.

Una de las novedades de mayor interés es la introducción de una serie de nuevos derechos de la era digital, así como sus garantías. Así, la LODPGDG parte de que los derechos y libertades que consagran la CE, así como los Tratados y Convenios Internacionales que los que es parte España son plenamente aplicables a Internet, debiendo contribuir los prestadores de servicios de internet y de la sociedad de la información a garantizar su aplicación (art. 79). En particular se concreta la regulación de un derecho a la seguridad digital (art. 82), el derecho a la educación digital, que dispone que el sistema educativo garantizará la plena inserción del alumnado en la sociedad digital (art. 83), protección de los menores en internet (art. 84), derecho de rectificación en internet (art. 85), el derecho a la actualización de informaciones en medios de comunicación digitales (art. 86), el derecho a la intimidad y uso de dispositivos digitales en el ámbito laboral (art. 87), el derecho a la desconexión digital en el ámbito laboral a fin de garantizar el respeto a su tiempo de descanso, permisos y vacaciones así como su intimidad (art. 88), el derecho a la intimidad frente al uso de dispositivos de videovigilancia y de grabación de sonidos en el lugar de trabajo (art. 89), el derecho a la intimidad ante la utilización de sistemas de geolocalización en el ámbito laboral (art. 90), los derechos digitales en la negociación colectiva (art. 91), la protección de datos de los menores en Internet (art. 92), el derecho al olvido en búsquedas de Internet (art. 93), derecho al olvido en servicios de redes sociales y servicios equivalentes (art. 94), derecho de portabilidad en servicios de redes sociales y servicios equivalentes (art. 95), derecho al testamento digital (art. 96), políticas de impulso de los derechos digitales (art. 97).

La LODPGDG contenía en la Disposición Final Tercera la modificación de la Ley Orgánica 5/1985, de 19 de junio, del Régimen Electoral General, que introducía un nuevo artículo 58bis sobre "Utilización de medios tecnológicos y datos personales en las actividades electorales" y que amparaba a los partidos políticos -sobre la base del interés público- (apartado primero) para que pudieran recopilar datos personales relativos a opiniones políticas de las personas, de tal forma que se podrían utilizar datos sobre opiniones políticas obtenidos en la web para el envío de propaganda personalizada ${ }^{26}$; esta posibilidad

\footnotetext{
${ }^{25}$ Respecto de la introducción en España del RGPD a través de la Ley Orgánica 3/2018, véase Ragone, Sarrión Esteve, et all)

${ }^{26}$ Artículo cincuenta y ocho bis. Utilización de medios tecnológicos y datos personales en las actividades electorales.

"1. La recopilación de datos personales relativos a las opiniones políticas de las personas que lleven a cabo los partidos políticos en el marco de sus actividades electorales se encontrará amparada en el interés público únicamente cuando se ofrezcan garantías adecuadas. [apartado declarado inconstitucional y nulo]

2. Los partidos políticos, coaliciones y agrupaciones electorales podrán utilizar datos personales obtenidos en páginas web y otras fuentes de acceso público para la realización de actividades políticas durante el periodo electoral.
} 
suscitó dudas de constitucionalidad que motivaron que el Defensor del Pueblo interpusiera un recurso de inconstitucionalidad el 4 de marzo de 2019, que ha sido estimado por el Tribunal Constitucional, que ha declarado en Sentencia 76/2019, de 22 de mayo, la inconstitucionalidad y nulidad del apartado 1 del precepto.

La nulidad de este apartado, teniendo en consideración la redacción del resto del artículo, no impide que los partidos políticos utilicen datos personales de webs y otras fuentes para la realización de actividades políticas (apartado 2), o la utilización de propaganda electoral por medios electrónicos (apartado 3), pero sí es cierto que deja de dar cobertura jurídica fundamentada en el interés público para realizarlo, por lo que sería necesario el consentimiento del interesado para que sus datos sean utilizados en campañas personalizadas.

De hecho, y como hemos apuntado antes, uno de esos retos digitales es la utilización de los smartphones, esos compañeros de viaje que llevamos a todas partes, y que permiten nuestro acceso rápido e inmediato, en tiempo real, a las noticias, informaciones, convocatorias, reuniones, etc.; pero que también sirven para nuestra geolocaización y obtener información de nuestros hábitos y costumbres, y ello no sólo con fines de investigación policial (para los que ya están siendo utilizados a través de la geolocalización temporal de los móviles en diversos casos) tanto respecto de víctimas como también respecto a presuntos responsables de delitos; sino también con otros fines como con fines de regulación laboral; fines estadísticos (como el reciente estudio que comenzó a realizar el Instituto Nacional de Estadística $^{27}$ ), y que fue permitido por la Agencia Española de Protección de Datos); o con fines de protección de la salud, como la que se ha planteado recientemente, a propósito del Radar COVID, a través de la Orden SND/297/2020, de 27 de marzo, por la que se encomienda a la Secretaría de Estado de Digitalización e Inteligencia Artificial, del Ministerio de Asuntos Económicos y Transformación Digital, el desarrollo de diversas actuaciones para la gestión de la crisis sanitaria ocasionada por el COVID-19, que antes hemos citado, y que incluyen el desarrollo de soluciones tecnológicas y aplicaciones móviles para la recopilación de datos para la mejora de la eficiencia operativa de los servicios sanitarios y una mejor atención y accesibilidad a los mismos por los ciudadanos, así como también encomendar un estudio de la movilidad aplicada a la crisis sanitaria (DataCOVID19), todo ello sin perjuicio de la aplicación del régimen de protección de datos ${ }^{28}$.

Otra de las grandes novedades a nivel interno es la elaboración de la Carta de Derechos Digitales, que se sometió a consulta pública a finales de 2020, y que elaborada por un grupo de expertos elaborada por el Grupo de Expertos constituido por la Secretaría de Estado de Digitalización e Inteligencia Artificial (SEDIA) del Ministerio de Asuntos Económicos y Transformación Digital, recoge un conjunto de principios y derechos para guiar los

3. El envío de propaganda electoral por medios electrónicos o sistemas de mensajería y la contratación de propaganda electoral en redes sociales o medios equivalentes no tendrán la consideración de actividad o comunicación comercial.

4. Las actividades divulgativas anteriormente referidas identificarán de modo destacado su naturaleza electoral. 5. Se facilitará al destinatario un modo sencillo y gratuito de ejercicio del derecho de oposición."

${ }^{27}$ Véase El INE comienza hoy a rastrear millones de móviles para conocer la movilidad de los españoles (2019).

${ }^{28}$ Sobre esta cuestión, véase Martínez Martínez (2020). 
proyectos normativos y las políticas públicas, para garantizar la protección de los derechos, tanto individuales como colectivos, en los entornos digitales que se abren. Esta Carta, atiende de forma especial al derecho a la igualdad en los entornos digitales, así como a la perspectiva de género en la transformación digita ${ }^{29}$.

En realidad, ahora mismo hay más interrogantes que respuestas, pero lo que es cierto es que quizá necesitemos un nuevo paradigma para responder a los retos de las tecnologías inteligentes desde la perspectiva de la protección de los derechos fundamentales, también respecto de la participación política por medios electrónicos o la e-participación, lo que requiere una profunda reflexión por parte de los estudiosos del Derecho Constitucional, también por supuesto en relación particular con el derecho de participación política, con la e-participación: ¿estamos ante una nueva dimensión del derecho de participación política? $¿$ o acaso es un nuevo derecho diferente?

La e-participación, en mi opinión, no es un derecho nuevo, sino una dimensión nueva de la participación política que se desarrolla a través de procedimientos electrónicos. Debe tenerse en cuenta que la participación puede ser activa y pasiva, es decir, en el sentido de que los ciudadanos deben poder participar activamente expresando su opinión, pero también tienen derecho a recibir de una forma transparente la información del proceso político, del debate en la esfera pública, es la única manera de que la democracia se sustente.

Uno de los peligros de la e-participación desde un punto de vista de la democracia constitucional no es tanto un debate activo en la nueva ágora virtual, sino la fragmentación de la misma en diferentes sectores, efecto de la polarización en la que las sociedades actuales se mueven ${ }^{30}$, junto con campañas específicas que pretende influir en los ciudadanos de una manera generalizada y no siempre muy bien intencionada con informaciones sesgadas.

Estos peligros están tan presentes en el ámbito urbano como el rural, al fin y al cabo, el mundo tecnológico o, mejor dicho, lo que se puede llamar el ambiente tecnológico inteligente (smart environments o ambient intelligence) que transforman la vida, y hacen cada vez más artificial -valga el juego de palabras- la distinción entre el mundo virtual y el real, por eso se puede hablar de onlife world (Hildebrandt, 2016: 8). Y si bien afecta a todos los ciudadanos, quizá en el mundo rural es más importante garantizar el acceso a los medios electrónicos y la nueva ágora virtual, porque de otra forma no podríamos contar con una importante voz que debe contribuir también al debate público en la democracia, por eso la e-democracia rural es tan importante; pero sin perder de vista, por supuesto, los propios peligros de la utilización de las tecnologías inteligentes que hemos anticipado, esto es, fundamentalmente el peligro de que no seamos nosotros mismos quienes participemos, sino que vengamos dirigidos, influenciados o determinados por otros cuando lo hacemos.

\footnotetext{
${ }^{29}$ Se puede acceder a la información sobre la consulta pública de la Carta de Derechos Digitales (SEDIA, 2020) aquí: https://www.mptfp.gob.es/portal/funcionpublica/secretaria-general-de-funcionpublica/Actualidad/2020/11/2020-11-19.html (Último acceso 07.05.2021).

${ }^{30}$ Ezra Klein explica en un reciente libro las razones de la polarización de la sociedad estadounidense. (Klein, 2020). Pero la sociedad europea, y la sociedad española también pueden verse reflejadas en esa polarización de identidades, donde la identidad del grupo es cada vez más relevante y determinante de las opiniones políticas, y de lo que seguimos, lo que escuchamos, y la esfera social reducida en la que tendemos a convivir de una forma cerrada.
} 
Rurali: Revista interdisciplinar de estudios rurales, N. 0, V.1, enero a junio de 2021, pp.18-41

\section{La e-participación en las redes: del estatus de consumidor al estatus de ciudadano}

En la práctica, la participación política a través de medios electrónicos o digitales, a través del mundo online, se manifiesta a través, fundamentalmente, de las redes tanto electrónicas como sociales. Es en este mundo donde las personas, los ciudadanos, convergemos y participamos, bien recibiendo, bien aportando todo lo que serían ideas, información, debatiendo, discutiendo, proponiendo, manifestando nuestras quejas, deseos, testimonios. Esto se hace en ocasiones de forma identificada y en otras ocasiones de forma anónima.

Hay varias cuestiones a tener en consideración. Por un lado, estas redes son de carácter privado, regidas por compañías extranjeras y que en general tienen unos fines concretos, con políticas que el usuario debe aceptar para poder entrar, crear su perfil y comenzar a comunicase con las mismas. Da igual que hablemos de unas redes o de otras, pues todas siguen en general esta idea. La cuestión es que el usuario de estas redes no es para la empresa proveedora del servicio un ciudadano sino un usuario, es decir, un consumidor; esto tiene, por supuesto, consecuencias desde el punto de vista del Derecho, y, por tanto, también para de los derechos que el mismo tiene en relación a estos servicios.

Un consumidor tiene una serie de derechos determinados por el estatus de consumidor, mientras que un ciudadano tiene una serie de derechos determinado por ese estatus, no es una diferencia baladí, pues el estatus determina la condición jurídica del titular, y el haz de derechos que puede ejercer. Un consumidor no tiene dentro del haz de derechos conferido el derecho de participación política, y esto porque como consumidor o usuario de un servicio, los derechos que tiene están relacionados con el servicio contratado, sea éste gratuito o previo pago, aunque en realidad siempre hay un pago aunque éste sea en datos, como ocurre en las redes.

Pues bien, debemos superar esta concepción. En el mundo híbrido en el que vivimos, donde ya vivimos más tiempo en el ambiente o mundo online que en el offline o real, los derechos de ciudadanía comenzamos a ejercerlos más en el mundo virtual que en el real, y el Derecho no puede renunciar a una regulación del mundo virtual que garantice una esfera pública que garantice que aquellos ciudadanos que quieren participar pueden participar.

De otra forma la e-participación, es decir, la participación política online o virtual no es real, sino que es un mero consumo de aquello que las propias plataformas permiten o consienten. Con esto no estamos diciendo que no haya que tener en consideración eventuales campañas de desinformación o de influencia sobre los ciudadanos, como siempre han existido en el mundo real, a través de medios más tradicionales. Desde 2018 la Unión Europea está muy preocupada en el mundo real por estas campañas de desinformación en el mundo virtual que afectan al proceso democrático en el mundo real.

El Grupo de Expertos de Alto Nivel sobre Noticias Falsas y Desinformación, designado por la Comisión Europea, recomienda que se exija una mayor transparencia a las plataformas y redes digitales, a la vez que se abandona la utilización del concepto de "noticia falsa", poco preciso, y se utiliza mejor el de "desinformación", y recomienda promover el desarrollo de herramientas que permitan desenmascarar la desinformación, salvaguardar la diversidad y estudiar el impacto que estas campañas pueden tener en Europa, además de un código de buenas prácticas a seguir por las plataformas y redes (Comisión Europea, 2018c); 
y la Comisión ha adoptado un plan de acción para enfrentarse a estas campañas de desinformación (Comisión Europea, 2018d), y que llevó a acordar junto con los representantes de plataformas, redes y empresas de publicidad a adoptar un Código de Buenas Prácticas sobre desinformación online y noticias falsas, en el que los signatarios (inicialmente Facebook, Google, Twitter y Mozilla junto con sectores de la industria de publicidad, Microsoft que se une en Mayo de 2019 y Tik Tok en Junio de 2020) se comprometen a adoptar políticas de lucha contra la desinformación y las noticias falsas ${ }^{31}$.

Es interesante tener en consideración, a efectos de este trabajo, que el Código de Buenas Prácticas tiene también un Anexo de Buenas Prácticas con enlaces a las políticas adoptadas por los signatarios.

Ciertamente la desinformación puede afectar a la calidad de la información que el ciudadano recibe, e incluso determinar e influir en su comportamiento y sus decisiones en el proceso participativo democrático; lo que hace comprensible la preocupación, o incluso el temor de la Unión Europea y de los actores que participan en el mundo digital de que esta desinformación afecte a la democracia digital ${ }^{32}$.

Pero la otra cara de la moneda es que políticas estrictas por parte de los actores en el mundo digital pueden limitar la participación política de los ciudadanos en el mundo online, en las redes, y por tanto, la e-participación. La e-participación no puede limitarse a recibir información, sino que debe también contemplar la participación activa a través de la manifestación de opiniones, dentro de unos límites, como en todo; lo complicado es delimitar la fina línea que separa una opinión de una información, pues la opinión salvo que atente contra la dignidad y sea susceptible de responsabilidad penal o civil no debería conllevar un control por parte de las plataformas, a diferencia de la (des)información. Pero la diferencia puede ser muy sutil.

Ello hace deseable que más allá del Código de Buenas Prácticas, las plataformas y redes no sean, en última instancia, quien decide qué es o no desinformación, a la hora de bloquear o censurar la e-participación, y quizá debería crearse algún tipo de organismo público que permita al usuario, que no deja de ser un ciudadano, acudir cuando no está conforme con la decisión de la plataforma, más allá de la posibilidad tortuosa de acudir a los tribunales; un organismo de este tipo, de carácter independiente, podría ser una solución.

\section{El gobierno inteligente y la e-participación}

La participación política online, la e-participación, va a depender también en gran medida de las iniciativas de la Administración Pública, y por tanto de las estrategias e implementación del gobierno inteligente (Smart governance), facilitando a través de los medios electrónicos de información (transparencia), como también del desarrollo de mecanismos de participación ciudadana, que pasa necesariamente por una transformación de la web de las distintas administraciones, que tiene un papel central en la "gestión pública del siglo XXI" (Santiago Iglesias, 2018: 418-419).

\footnotetext{
${ }^{31}$ Véase el Código de buenas prácticas de la Unión en materia de desinformación (2018).

${ }^{32}$ Véase, entre otros, Dutton (2018), Llanera González (2018), Parreira Prado (2019)..
} 
Esto, por supuesto, se puede dar a todos los niveles, así podemos ver como en la Unión Europea encontramos de forma regular iniciativas de consulta ciudadana sobre las políticas de la Unión Europea, a nivel interno podemos ver el intento de potenciar la transparencia por parte de la Administración Pública, a través del Portal de Transparencia. No obstante, es a nivel local o municipal donde se pueden identificar las iniciativas más desarrolladas en este contexto, quizá porque la política municipal es la más próxima al ciudadano.

No obstante, en el proyecto Mapping Smart Cities in the EU (Parlamento Europeo, 2014) se toman en consideración las ciudades de más de 100.000 habitantes, relegando a las ciudades pequeñas y al mundo rural. ¿Acaso no son importantes las ciudades pequeñas, o los pequeños municipios y villas? También son importantes, y parece que los objetivos de las Smart cities les serían aplicables: crear ciudadanos empoderados y que se conecten en la comunidad inteligente, con iniciativas bottom-up, inspirar líderes, aproximaciones holísticas y participativas a través de la colaboración, el codesarrollo; y se pone como ejemplos la Smart City Barcelona, Copenaghe, Viena, Ámsterdam, etc..

El Plan Nacional de Ciudades Inteligentes (2015-2017) perseguía precisamente mejorar la prestación de los servicios públicos por parte de las entidades locales gracias al uso de las TIC, mejorando también su sistema de gobernanza con la participación de distintos actores $^{33}$, y en las distintas convocatorias ha permitido la financiación de proyectos de como ciudades inteligentes de Alcalá la Real, Costa del Sol, Granada, Huelva, Lepe, Martos, Sevilla, Toledo, Valdepeñas, Almendralejo-Badajoz, Villanueva de la Serena (1 ${ }^{\mathrm{a}}$ convocatoria con 15 millones de euros), Alicante, Cáceres, Córdoba, Gijón, Las Palmas de Gran Canaria, Lugo, Murcia, Palencia, Zaragoza-Madrid-A Coruña-Santiago de Compostela, Ponferrada, Santander, Valencia, Valladolid, Segovia ( $2^{\mathrm{a}}$ convocatoria con 63 millones de euros), y las islas de El Hierro, Fuerteventura y Mallorca (Convocatoria Islas Inteligentes, con 30 millones de euros).

Es con el Plan de Territorios Inteligentes (2017-2020), donde comienza a subrayarse la relevancia de mejorar los servicios y plataformas tecnológicas tanto de la ciudad como del mundo rural ${ }^{34}$.

Desde la Administración Pública es importante poner el foco en la potenciación del mundo rural por varias razones, pero principalmente porque el despoblamiento y vaciamiento de lo rural hace más difícil de forma progresiva su potenciación por sí mismo; es esencial no solo garantizar unos servicios mínimos (en educación, sanidad, redes de internet incluyendo 5G), sino también canalizar instrumentos que permitan que sus ciudadanos sean activos en la e-participación; para esto el papel de la Administración es esencial, pues como se ha señalado con acierto "es la Administración quien, finalmente, tiene la decisión de lo que se hace con el espacio público", y puede ser determinante potenciar que los pequeños núcleos de población sean ciudades educadoras, e inteligentes, que potencien la cultura teniendo en consideración la realidad en su totalidad con sus potencialidades (Benlloch Domènech, Benítez Giralt, 2020).

\footnotetext{
${ }^{33}$ Plan Nacional de Ciudades Inteligentes (2015-2017).

${ }^{34}$ Plan de Territorios Inteligentes (2017-2020).
} 


\section{A modo de conclusión: ¿Hacia una democracia digital?}

Desde hace mucho tiempo vivimos en una democracia de partidos, la democracia representativa propia del régimen constitucional liberal ha mutado y transformado la democracia actual en una democracia en la que los partidos políticos son protagonistas del proceso político, de forma que los instrumentos de democracia directa (referendo, iniciativa legislativa popular) no son sino complementos que, como muy bien dice A. Torres del Moral, se han visto en muchas ocasiones con cierto recelo, pues en épocas pasadas sirvieron de apoyo a regímenes autoritarios, aunque se han introducido en las Constituciones contemporáneas (Torres del Moral, 2015: 409 y ss.). Hoy en día, los políticos siguen reacios en muchas ocasiones a utilizar estos mecanismos, y cuando lo hacen -cuando no es obligatorio hacerlo- es porque piensan que el resultado les va a favorecer aun cuando siempre sea imprevisible, como el referéndum del Brexit demostró no hace tanto tiempo

Cabe preguntarse si la evolución y revolución tecnológica con las mayores facilidades de participación ciudadana pueden cambiar el modelo o incluso lo está cambiando ya hacia una democracia digital.

Es cierto que el voto electrónico - a distancia- a día de hoy no parece tan seguro como el voto tradicional, y esto motiva que haya suspicacias en relación con la seguridad de la encriptación del proceso de voto, la protección de los datos de los electores, o el posible control y auditoría posterior que permita procesos de participación seguros, en definitiva, lo que se podrían considerar como los principios que rigen un proceso electoral tradicional (la libertad de sufragio, igualdad en el derecho de voto, el secreto, etc.) ${ }^{35}$.

Ahora bien, una cosa es la participación política de carácter consultivo en ciertas decisiones, o incluso resolutiva respecto de elecciones, y otra cosa distinta es la participación activa en el debate público que influye en las deciones de los representantes y de los propios partidos políticos.

En general lo que está ocurriendo es que hay una mayor inmediatez en todo debido al mundo digital, y que la democracia actual no ha cambiado en su sustancia aunque sí respecto al proceso comunicativo con los ciudadanos, ahí es donde está la diferencia dada la tecnología que permite una comunicación prácticamente instantánea y casi directa con el ciudadano a través de las redes y plataformas, que no necesariamente debe afectar a la esencia y contenido prescriptivo del régimen constitucional democrático.

Se suele decir que, conforme a lo previsto en la Declaración de Derechos del Hombre y del Ciudadano de 1789 que "una sociedad en la que no esté establecida la garantía de los derechos, ni determinada la separación de los poderes, carece de constitución", lo que unido a la incorporación de la dimensión social en la configuración del Estado define nuestro modelo como un Estado social y democrático de Derecho (art. 1.1 CE).

La preocupación existente actualmente por el crecimiento de algunas corrientes iliberales en Europa y en otros lugares, en muchas ocasiones con cierto sustento y soporte académico (Brenan, 2018) e incluso popular, no puede hacernos olvidar los logros de la

\footnotetext{
${ }^{35}$ Sobre los problemas que se plantean en las experiencias con voto electrónico a distancia véase González de la Garza (2010).
} 
democracia constitucional, ni tampoco que la participación de los ciudadanos, en cuanto integrantes del pueblo soberano, es esencial para la propia esencia de la democracia.

De hecho, la participación del pueblo, de los ciudadanos, con las debidas garantías, en el debate público, es precisamente lo que puede salvar la democracia. El principio, manido muchas veces, de Salus Publica suprema lex esto, que se deriva del viejo principio proclamado por Cicerón "Regio imperio duo sunto, iique a praeeundo iudicando consulendo praetores iudices consules appellamino. Militae summum ius habento nemini parento. Ollis salus populi suprema lex esto" 36 , y que se suele utilizar con expresiones variantes (Salus publica suprema lex est, o salus publica suprema lex esto), en realidad no significa que la salud del pueblo es la ley suprema, pues el concepto sería más próximo al del bienestar del pueblo, que incluye también su salud; por eso, quizá, como ya hemos dicho en otro lugar (Sarrión Esteve, 2020c), la traducción al inglés de esta ley de Cicerón -the people's good is the highest law- es más precisa que la que utilizamos en español ${ }^{37}$.

Por ello, es importante perseguir el bienestar del pueblo, pero es difícil hacerlo sin darle voz, hay que perder el miedo a abrir y potenciar canales de participación política de los ciudadanos a través de las tecnologías digitales, para enriquecer el debate público, garantizando eso sí, los principios propios de una sociedad abierta y plural, y para ello, hay que garantizar que el mundo rural participa también en este debate y se garantiza su acceso a la e-participación.

\section{Recursos bibliográficos.}

Asís Roig, R. de (2015). Una mirada a la robótica desde los derechos humanos, Dykinson.

Banafa, A. (2019). Blokchain y la inteligencia artificial: ¿la pareja ideal?. OpenMind, 6/05/2019. https://www.bbvaopenmind.com/tecnologia/inteligencia-artificial/blockchain-yla-inteligencia-artificial-la-pareja-idealblockchain-y-la-inteligencia-artificial-la-parejaideal/ (Último acceso 14.09.2019).

Barrio Andrés, M. (2018) (dir). Derecho de los robots. Wolters Kluwer.

Barrio Andrés, M. (2020). Manual de Derecho Digital, Tirant lo blanch.

Benlloch Domènech, C., y Benítez Giralt, R. (2020). La «Ciudad Educadora» como herramienta de empoderamiento rural". Blog CEPC, 9.06.2020. http://www.cepc.gob.es/cepc/blog/blog_cepc/2020/06/09/la-ciudad-educadora-comoherramienta-de-empoderamiento-rural (Último Acceso 20.09.2020).

Brenan J. (2018). Contra la Democracia, Deusto.

Burguera Ameave, L. (2014). Identidad política y participación: la iniciativa ciudadana europea, Estudios de Deusto, 62, 2, 391-403.

Cambridge Analytica: la multa récord que deberá pagar Facebook por la forma en que manejó los datos de 87 millones de usuarios (2019). BBC News Mundo Redacción. https://www.bbc.com/mundo/noticias-49093124 (Último acceso 19.09.2020).

\footnotetext{
${ }^{36}$ Citado en Sánchez de la Torre (1997).

37 Véase al respecto Hawson (2002).
} 
Christensen, B. (26 Mayo 2006). Asimov's First Law: Japan Sets Rules for Robots. Live Science. $\quad$ http://www.livescience.com/10478-asimov-law-japan-sets-rules-robots.html (último acceso 23.01.2021).

Código de buenas prácticas de la Unión en materia de desinformación (2018). CNECT-201920022-00-00-ES-TRA. $\quad$ https://ec.europa.eu/digital-single-market/en/code-practicedisinformation (Último Acceso 13.02.2021).

Comisión Europea (2018a). Comunicación de la Comisión "Inteligencia artificial para Europa". SWD (2018) 137 final. 25.4.2018. https://eur-lex.europa.eu/legalcontent/ES/TXT/HTML/?uri=CELEX:52018DC0237\&from=ES (último acceso 23.01.2021).

Comisión Europea (2018b). Propuesta de Reglamento por el que se establece el programa Europa Digital para el período 2021-2027. 2018/0227/COD, 2020. https://eurlex.europa.eu/legal-content/ES/HIS/?uri=CELEX:52018PC0434 (Último acceso 30.01.2021).

Comisión Europea (2018c). A multi-dimensional approach to disinformation. Report of the independent High level Group on fake news and online disinformation. http://ec.europa.eu/newsroom/dae/document.cfm?doc_id=50271 (Último acceso 20.09.2020).

Comisión Europea (2018d). Joint Communication to the European Parliament, the European Council, the Council, The European Economic and Social Committee and the Committee of the Regions. Action Plan Against Disinformation. 5.12.2018, JOIN(2018) 36 final. https://ec.europa.eu/digital-single-market/en/news/action-plan-against-disinformation (Último Acceso 20.09.2020).

Comisión Europea (2020a). LIBRO BLANCO sobre la inteligencia artificial - un enfoque europeo orientado a la excelencia y la confianza, 19 de febrero de 2020. https://ec.europa.eu/info/sites/info/files/commission-white-paper-artificial-intelligencefeb2020_es.pdf (Último acceso 30.01.2021).

Comisión Europea (2020b). Informe sobre las repercusiones en materia de seguridad y responsabilidad civil de la inteligencia artificial, el internet d elas cosas y la robótica. COM/2020/64 final. https://eur-lex.europa.eu/legalcontent/ES/TXT/?qid=1593079180383\&uri=CELEX:52020DC0064 (Último acceso 30.01.2021).

Comisión Europea (2021). Proposal for a Regulation of the European Parliament and of the Council laying down harmonized rules on Artificial Intelligence (Artificial Intelligence Act) and amending certain Union legislative acts. 2021/0106 (COD). https://digitalstrategy.ec.europa.eu/en/library/proposal-regulation-laying-down-harmonised-rulesartificial-intelligence-artificial-intelligence (Último acceso 06.05.2021).

Consejo de Europa (2017a). Recommendation 2102. Technological convergence, artificial intelligence and human rights. Committee on Legal Affairs and Human Rights. http://assembly.coe.int/nw/xml/XRef/Xref-DocDetails-EN.asp?FileID=23726\&lang=EN

(Último acceso 30.01.2021).

Consejo de Europa (2017b). Reply to Recommendation R2102. Committee of Minister. Doc. 14432. 19 Octubre 2017. http://assembly.coe.int/nw/xml/XRef/Xref-DocDetailsEN.asp?FileID=24236\&lang=EN (Último acceso 30.01.2021). 
Consejo de Europa (2018). European Commission for the Efficiency of Justice (CEPEJ). European ethical Charter on the use of Artificial Intelligence in judicial systems and their environment. Adoptada en sesión plenaria en Estrasburgo 3-4 Diciembre de 2018. https://rm.coe.int/ethical-charter-en-for-publication-4-december-2018/16808f699c (Último acceso 18.03.2020).

Cotino Hueso, L. (2017). Big data e inteligencia artificial. Una aproximación a su tratamiento jurídico desde los derechos fundamentales. ILEMATA, 24,131-150.

Craglia, M. (2018) (Ed.). Artificial Intelligence- A European Perspective. Publications Office.

DAIA (2019). Conclusiones del I Seminario de Internacional Derecho Administrativo e Inteligencia Artificial. https://www.um.es/idertec/wpcontent/uploads/2019/04/CONCLUSIONES-SEMINARIO-DAIA.vfinal.pdf (Último acceso 19.09.2020).

Darling, K. (2016). Extending Legal Protection to Social Robots: The Effects of Anthropomorphism, Empathy, and Violent Behavior Towards Robotic Objects. Robot Law, Calo, Froomkin, Kerr eds., Edward Elgar 2016, We Robot Conference 2012, University of Miami. SSRN: https://ssrn.com/abstract=2044797 (Último acceso 14.09.2019).

Déaut Le, D. (2017). Technological convergence, artificial intelligence and human rights. Council of Europe, Committee on Culture, Science, Education and Media, Report Doc. $1428810 \quad$ April 2017. http://assembly.coe.int/nw/xml/XRef/Xref-DocDetailsEN.asp?FileID=23531\&lang=EN (Último acceso 14.09.2019).

Dutton, W. H. (2018). ¿Son las “fake news” una fake new?. Temas para el debate, 278-279, 26-28.

El INE comienza hoy a rastrear millones de móviles para conocer la movilidad de los españoles (2019). RTVE 18/11/2019. https://www.rtve.es/noticias/20191118/ine-comienzahoy-rastrear-millones-moviles-para-conocer-movilidad-espanoles/1991280.shtml(Último acceso 04.04.2020).

García Novoa, C., D. Santiago Iglesias, (2018) (dir). $4^{a}$ Revolución industrial: Impacto de la automatización y la inteligencia artificial en la sociedad y la economía digital. Thomsom Reuters Aranzadi.

Gómez Sánchez, Y. (2013). La iniciativa Ciudadana en la Unión Europea. Panorama Social, 17, 59-69.

Gómez Sánchez, Y. (2018). Constitucionalismo Multinivel. Derechos Fundamentales. $4^{\mathrm{a}}$ edición. Sanz y Torres.

González de la Garza, L. M. (2010). Voto electrónico por internet y riesgos para la democracia (II). Revista de Derecho Político, 77, 213-249.

Gonzalo, M. (2020). Un centenar de académicos piden al Gobierno más transparencia en el desarrollo de Radar COVID. Newtral, 05/09/2020. https://www.newtral.es/comunidadacademica-publica-manifiesto-transparencia-radar-covid/20200905/ (Último acceso 19.09.2020).

Grupo Independiente de Expertos de Alto Nivel sobre Inteligencia Artificial de la Comisión Europea (2019). Directrices Éticas para una IA fiable. https://ec.europa.eu/futurium/en/aialliance-consultation (último acceso 30.01.2021). 
Hawson, H. (2002). Unwritten Laws: the unofficial rules of life as handed down by Murphy and other sages. Castle Books.

Hildebrandt, M. (2016). Smart technologies and the End(s) of Law. Edwar Elgar Publishing. Huergo Lora, A. (2021). El proyecto de Reglamento sobre la Inteligencia Artificial. Almacén del Derecho, https://almacendederecho.org/el-proyecto-de-reglamento-sobre-lainteligencia-artificial (Último acceso 06.05.2021).

Klein, E. (2020). Why We're Polarized. Avid Reader Press.

López Baroni, M. (2019). Las narrativas de la inteligencia artificial. Revista de Bioética y Derecho, 46.

Lumpkin, D. (2013). Are robots the future of sex?. Jincey Lumpkin Esq. at TEDxSiliconAlley. Videotalk, https://www.youtube.com/watch?v=nxVBjfHzdI4 (Último acceso 14.09.2019).

Llanera González, P. (2018). Fake news, monopolio, y democracia: Cómo controlar el poder de los GAFA. Revista de privacidad y derecho digital, 3 (11), 77-107.

Martínez Martínez, R. (2020). Protección de datos y geolocalización en la Orden SND/297/2020. HayDerecho-Expansión. 31/03/2020. https://hayderecho.expansion.com/2020/03/31/proteccion-de-datos-y-localizacion-en-laorden-snd-297-2020/ ( Último acceso 03.04.2020).

Ministerio de Ciencia, Innovación y Universidades (2019). Estrategia Española de $I+D+I$ en Inteligencia Artificial. http://www.ciencia.gob.es/stfls/MICINN/Ciencia/Ficheros/Estrategia_Inteligencia_Artifici al_IDI.pdf (último acceso 30.01.2021).

Parlamento Europeo (2014). Mapping Smart Cities in the EU. https://www.europarl.europa.eu/RegData/etudes/etudes/join/2014/507480/IPOLITRE_ET(2014)507480_EN.pdf (Último Acceso 20.09.2020).

Parlamento Europeo (2017). Resolución del Parlamento Europeo, de 16 de febrero de 2017, con recomendaciones destinadas a la Comisión sobre normas de Derecho civil sobre robótica (2015/2103(INL)). $\quad$ https://www.europarl.europa.eu/doceo/document/TA-8-20170051_ES.html (Último acceso 23.01.2021).

Parlamento Europeo (2019). Resolución de 12 de febrero de 2019 sobre "Una política industrial global europea en materia de inteligencia artificial y robótica". A8-0019/2019. http://www.europarl.europa.eu/sides/getDoc.do?pubRef=-//EP//TEXT+TA+P8-TA-20190081+0+DOC+XML+V0//ES (Último acceso 23.01.2021).

Parreira Prado, M. (2019). La proliferación de las "fake news" y sus algoritmos daña la cultura democrática. Ambitos: Revista internacional de comunicación, 45, 89-106.

Pérez Zafrilla, P. J.; Benlloch Domènech, C.; Sarrión Esteve, J. (2021). e-Democracia rural. Tirant lo blanch.

Plan de Territorios Inteligentes (2017-2020).: https://www.red.es/redes/es/quehacemos/territorios-inteligentes (Último Acceso 20.09.2020).

Plan Nacional de Ciudades Inteligentes (2015-2017). https://www.red.es/redes/es/quehacemos/ciudades-inteligentes/plan-nacional-de-ciudades-inteligentes (

Pollock, D. (2018). The Fourth Industrial Revolution Built On Blockchain and Advanced with AI. Forbes, 30/11/2018. https://www.forbes.com/sites/darrynpollock/2018/11/30/the- 
Rurali: Revista interdisciplinar de estudios rurales, N. 0, V.1, enero a junio de 2021, pp.18-41

fourth-industrial-revolution-built-on-blockchain-and-advanced-with-ai/\#3e3cc0ef4242

(Último acceso 14.09.2019)

Ragone, S.; Sarrión Esteve, J; Wisschmeyer, T.; Woods, L. (2019). Appendice comparata. En Il "nuevo" codige in materia di protezione dei dati personali (319-344). Giappicheli.

Replicante Legal (2014). ¿Pueden tener derechos los robots?”, 2014. http://replicantelegal.com/pueden-tener-derechos-los-robots/ (Último acceso 14.09.2019).

Sánchez de la Torre, A. (1997). El objeto de la legalidad en la expresión salus populi suprema lex esto. Cuadernos de filología clásica: Estudios latinos, 12, 39-78.

Sánchez Barrilao, J. F. (2016). El Derecho Constitucional ante la era de Ultrón: la informática y la inteligencia artificial como objeto constitucional. Estudios de Deusto, 64/2, 225-258.

Santiago Iglesias, D. (2018). «Smart cities»: aproximación a un fenómeno en auge. En $4^{a}$ Revolución Industrial: impacto de la automatización y la inteligencia artificial en la sociedad y la economía digital (413-433). Thomsom Reuters Aranzadi.

Sarrión Esteve, J. (2018). Epílogo. Actuales retos para la regulación de los drones. En M. del Barrio Andrés (Dir.). Derecho de los drones, (389-398). La Ley Wolter Wluver.

Sarrión Esteve, J. (2020a). "El Derecho Constitucional en la era de la Inteligencia Artificial, los Robots y los Drones". En Setenta años de Constitución Italiana y cuarenta años de Constitución Española. 5, Retos en el siglo XXI (321-334). CEPC y BOE.

Sarrión Esteve, J. (2020b). Los Derechos Humanos ante la transformación digital y las tecnologías inteligentes: hacia un nuevo paradigma. En Los Derechos Humanos en el siglo XXI. En la conmemoración del 70 Aniversario de la Declaración, T III. Edisofer.

Sarrión Esteve, J. (2020c). Sobre el constitucionalismo del bien común en los tiempos del COVID: ¿es posible un constitucionalismo iliberal?. Blog CEPC, 11.05.2020. http://www.cepc.gob.es/cepc/blog/blog_cepc/2020/05/11/sobre-el-constitucionalismo-delbien-com\%C3\%BAn-en-los-tiempos-del-covid-es-posible-un-constitucionalismo-iliberal(Último Acceso 20.09.2020).

Sarrión Esteve, J., y Benlloch Domènech, C. (2017). Rights and science in the drone era. Actual challenges in the civil use of drone technology. Rights \& Science, 0, 117-133.

SEDIA. Secretaría de Estado de Digitalización e Inteligencia Artificial. (2020). Documento para consulta pública. Carta de Derechos Digitales. Accesible aquí: https://portal.mineco.gob.es/RecursosArticulo/mineco/ministerio/participacion_publica/au diencia/ficheros/SEDIACartaDerechosDigitales.pdf (Último Acceso 07.05.2021).

Torres del Moral, A. Estado de Derecho y Democracia de Partidos, $5^{\text {a }}$ edición. Universitas.

Valero Torrijos, J. (2019). Las garantías jurídicas de la inteligencia artificial en la actividad administrativa desde la perspectiva de la buena administración. Revista catalana de dret públic, 58, 82-96.

Vida Fernández, J. (2018). Los retos de la regulación de la Inteligencia Artificial: algunas aportaciones desde la perspectiva europea. En Sociedad Digital y Derecho. BOE.

Weller, C. (7 Julio 2016). Law firms of the future will be filled with robot lawyers. Business Insider. http://www.businessinsider.com/law-firms-are-starting-to-use-robot-lawyers-20167 (Último acceso 23.01.2021).

Wolfson, R. (2018). Diversifying Data With Artificial Intelligence and Blockchain Technology.

Forbes,

20/11/2018. 
Rurali: Revista interdisciplinar de estudios rurales, N. 0, V.1, enero a junio de 2021, pp.18-41

https://www.forbes.com/sites/rachelwolfson/2018/11/20/diversifying-data-with-artificialintelligence-and-blockchain-technology/\#1b3c010f4dad (Último acceso 14.09.2019) 